\title{
Electrophysiological properties of la excitation and recurrent inhibition in cat abdominal motoneurons
}

\author{
Masatoshi Niwa ${ }^{1} \cdot$ Ken Muramatsu ${ }^{2} \cdot$ Kiyomi Nakayama $^{3} \cdot$ Sei-Ichi Sasaki ${ }^{4,5}$
}

Received: 13 March 2018 / Accepted: 28 September 2018 / Published online: 15 October 2018

(C) The Physiological Society of Japan and Springer Japan KK, part of Springer Nature 2018

\begin{abstract}
Ia excitation and recurrent inhibition are basic neuronal circuits in motor control in hind limb. Renshaw cells receive synaptic inputs from axon collaterals of motoneurons and inhibit motoneurons and Ia inhibitory interneurons. It is important to know properties of Ia excitation and recurrent inhibition of trunk muscle such as abdominal muscles. The abdominal muscles have many roles and change those roles for different kind of functions. Intracellular recordings were obtained from the abdominal motoneurons of the upper lumbar segments in cats anesthetized. First, dorsal roots were left intact, and sensory and motor axons were electrically stimulated. Ia excitatory post-synaptic potentials were elicited in five of eight motoneurons at same segment stimulated. Second, dorsal roots were sectioned, and motor axons were electrically stimulated. Recurrent inhibitory post-synaptic potentials were elicited in one of 11 abdominal motoneurons. Renshaw cells extracellularly fired high-frequency bursts at short latency and at same segment stimulated.
\end{abstract}

Keywords Abdominal motoneuron $\cdot$ Ia-EPSP $\cdot$ Recurrent IPSP $\cdot$ Spinal cord $\cdot$ Inhibition

Masatoshi Niwa

mt-niwa@ks.kyorin-u.ac.jp

Ken Muramatsu

k.muramatsu@kenkoudai.ac.jp

Kiyomi Nakayama

nakayamak@dent.showa-u.ac.jp

Sei-Ichi Sasaki

sasakis@ipu.ac.jp

1 Department of Occupational Therapy, Kyorin University, 5-4-1 Shimorenjaku, Mitaka, Tokyo 181-8612, Japan

2 Department of Physical Therapy, Health Science University, 7187 Kodachi, Fujikawaguchiko, Yamanashi 401-0380, Japan

3 Department of Oral Physiology, School of Dentistry, Showa University, 1-5-8 Hatanodai, Shinagawa, Tokyo 142-8555, Japan

4 Center for Medical Sciences, Ibaraki Prefectural University of Health Sciences, 4669-2 Ami, Ami-machi, Inashiki, Ibaraki 300-0394, Japan

5 Present Address: Tokyo Public Health College, 6-21-7 Hommachi, Shibuya-ku, Tokyo 151-0071, Japan

\section{Introduction}

Recently, we reported that the patterns of neural discharge in the abdominal muscles are critical for increasing intraabdominal pressure and inducing defecation [1]. Moreover, the abdominal muscles play important roles in posture and walking [2, 3], vomiting [4], overall respiration, and the control of expiration $[5,6]$. The abdominal muscles include the external oblique (EO), internal oblique (IO), transverse abdominis (TA), and rectus abdominis (RA), which are innervated by motoneurons located in the ventral horn of the lower thoracic (T) and lumbar (L) spinal cord [7]. Previous studies have recorded the activity of alpha and gamma motor axons from the peripheral nerves of the abdominal muscles, demonstrating that positive pressure expiration is associated with alpha-gamma linkage [6].

Ia fibers convey monosynaptic excitation to motoneurons and are responsible for almost all monosynaptic excitatory postsynaptic potentials (EPSPs) elicited in the motoneurons of the hind limb [8-10] and intercostal muscles [11]. Stretching of the muscles spindles increases the discharge of Ia fibers, which then influence the activity of alpha motoneurons [8]. However, little is known regarding the effects of reflex actions on abdominal motoneurons. 
Recurrent inhibition, which was first observed in motoneurons of the hind limb, is thought to control motor output in the spinal cord [12]. Recurrent inhibition is mediated by Renshaw cells, which are excited via the axon collaterals of motoneurons due to stimulation of the ventral roots [13]. In the lumbosacral spinal cord, Renshaw cells inhibit not only alpha motoneurons but also Ia inhibitory interneurons $[29,30]$. It was assumed that Renshaw cells regulate the motor output of the agonist by recurrent inhibition and of the antagonist by Ia inhibitory interneurons in a parallel fashion. In addition to the hind limb muscles, recurrent inhibition has been observed in the intercostal muscles [14], neck muscles [15], and diaphragm [16]. However, Renshaw cells unlikely inhibit Ia inhibitory interneurons for intercostal motoneurons, since Ia reciprocal inhibition was not observed between the internal intercostal motoneurons and the external intercostal motoneurons [11]. Moreover, no Ia synaptic potentials were detected from phrenic motoneurons by stimulation of the ipsilateral phrenic nerve [17]. The abdominal muscles normally show the expiratory activities but have many roles during voluntary movements in awake animals [18]. However, the monosynaptic excitation from Ia fibers and recurrent inhibitory pathways affecting the motoneurons remain unknown. In the present study, we aimed to determine how monosynaptic excitation from Ia fibers and recurrent inhibitory pathways influence the activity of abdominal motoneurons in L1 and L2 spinal segments.

\section{Materials and methods}

\section{Ethical approval}

All experimental procedures were approved by the Animal Ethics Committee of Ibaraki Prefectural University of Health Sciences and were in accordance with the guiding principles for care and use of animals in the field of physiological sciences outlined by the Physiological Society of Japan.

\section{Surgical procedures}

The experiments of the present study involved ten adult cats (Shiraishi Animals, Japan) anesthetized with sodium pentobarbital (initial dose: $35-40 \mathrm{mg} / \mathrm{kg}$, i.p.). The cats were intubated with a tracheal tube, and tracheal pressure was monitored. The femoral artery and forearm vein were cannulated, and arterial blood pressure was maintained at 100-130 mmHg via intravenous administration of pressor agents (Nor-adrenalin, Daiichi-Sankyo, Japan), as needed. Rectal temperature was maintained at $37-38{ }^{\circ} \mathrm{C}$ using a heating pad. Deep anesthesia (narrow pupil size and stable arterial blood pressure) was subsequently maintained using supplemental doses of sodium pentobarbital throughout the experiments (4-7 mg/kg/h, i.v.).

The T13 and L1-L3 abdominal peripheral nerves were dissected free from the abdominal muscles. Bipolar cuff electrodes (inter-electrode distance of $2 \sim 3 \mathrm{~mm}$ ) were placed at the proximal portion of the whole nerves for electrical stimulation. The lateral branches of L1 and L 2 that innervate the EO muscle were dissected and sliced at the distal portion, just prior to their entrance into the EO muscle. The medial branches of L1 and L2 that innervate the IO, TA, and RA muscles were also dissected free from the surrounding tissue and sliced just prior to the insertion point (Fig. 1). The spinal cord was exposed via laminectomy at T12-L4 and opening of the dura mater. The edge of the dura mater was sewn to the erector spinae muscles with fine thread to ensure proper positioning of the spinal cord. Animals were immobilized via intravenous administration of pancuronium bromide ( $0.4 \mathrm{mg} / \mathrm{h}$, Mioblock, Sankyo. Organon) and maintained on artificial ventilation, which was adjusted to maintain an end-expired $\mathrm{CO}_{2}$ level of 4-6\%. To eliminate descending supraspinal input, the spinal cord was transected at the T12-T13 junction. Bilateral pneumothorax was induced to remove the influence of breathing in the spinal cord. A mineral oil pool was formed over the exposed region of the spinal cord. Two types of dorsal root preparations were used. In the first type of preparation $(N=3)$, the dorsal roots were left intact, and Ia-EPSPs were recorded from the abdominal motoneurons. In the second type of preparation $(N=7)$, the dorsal roots were cut, recurrent inhibitory postsynaptic potentials (R-IPSPs) were recorded from the abdominal motoneurons, and extracellular spikes were recorded from a single Renshaw cell.

\section{Recording procedures}

Glass micro-electrodes filled with $2 \mathrm{M}$ K-Citrate (20-30 M $\Omega$ ) or $3 \mathrm{M} \mathrm{KCl} \mathrm{(10-20} \mathrm{M \Omega )} \mathrm{were} \mathrm{used} \mathrm{to} \mathrm{obtain}$ intracellular recordings from abdominal motoneurons. The micro-electrodes were placed at a mediolateral angle of $5-20^{\circ}$ and inserted into the ventral region of the spinal cord through a hole in the pia mater. Nerves were stimulated with rectangular pulses of $150 \mu$ s in duration. Abdominal motoneurons were identified via antidromic activation following stimulation of the lateral or medial branches of L1 and L2. Only motoneurons with stable membrane potentials exceeding $-40 \mathrm{mV}$ and without spontaneous spike activity were selected for analysis.

Intracellular recordings were obtained from abdominal motoneurons in order to identify antidromic spikes and EPSPs following stimulation of the relevant abdominal peripheral nerves with intact dorsal roots. Afferent volleys arriving at the dorsal-root entry zone were recorded using a silver ball electrode. 


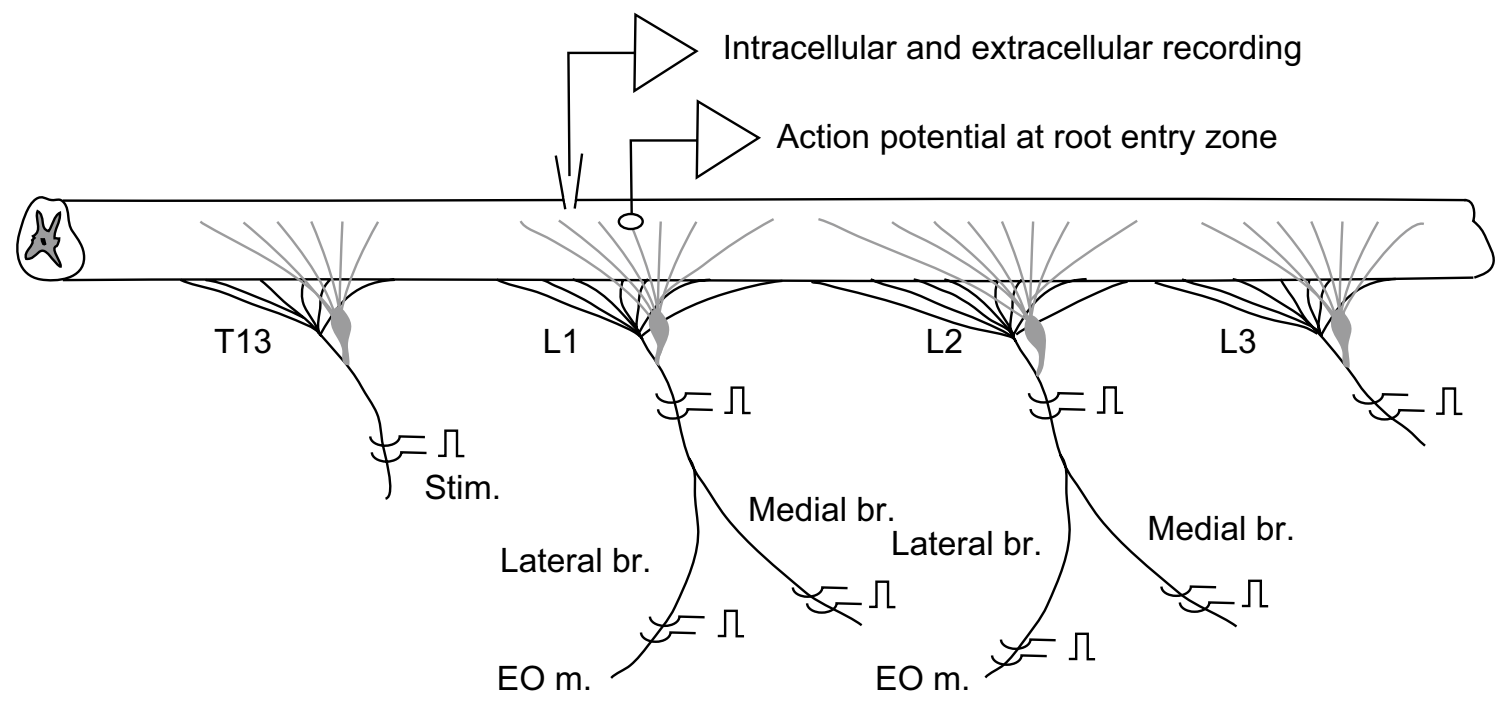

Fig. 1 Experimental arrangement. Intracellular recordings were obtained from abdominal motoneurons with intact dorsal roots in order to identify antidromic spikes and Ia-EPSPs. Afferent volleys arriving at the dorsal-root entry zone were recorded using silver ball electrodes. The dorsal roots were cut in order to record R-IPSPs from

After the ipsilateral T12-L4 dorsal roots had been cut to eliminate sensory input, intracellular recordings were obtained from abdominal motoneurons to identify R-IPSPs following stimulation of the relevant abdominal peripheral nerves. When stable intracellular recordings were obtained (filtering of DC $\sim 5 \mathrm{kHz}$ ), the stimulus voltage was gradually decreased until antidromic activation of the impaled motoneuron could no longer be elicited. Then, the membrane potentials were summed 50 to 200 times using a signal averaging system (VC-11 and QC-111 J, Nihonkohden, Japan), and stimulation was applied at a frequency of 2 or $2.5 \mathrm{~Hz}$.

Glass micropipettes filled $2 \mathrm{M} \mathrm{NaCl}$ solution saturated with Fast Green FCF dye (1-2 M $\Omega$ in resistance) were used to obtain extracellular recordings from single Renshaw cells. Renshaw cells were described as the interneurons that volleys of impulses in motor axons generate an inhibitory post-synaptic potential of the motoneuronal membrane and a prolonged repetitive discharge [12]. In the present study, neurons in which activity was evoked synaptically and the discharge was evoked over the same stimulus range as the antidromic field potential corresponding to the stimulus range of activation of alpha motor axons of abdominal nerve stimulation were regarded as Renshaw cells, since the dorsal roots had been cut at the corresponding spinal level [14]. Responses of Renshaw cells to stimulation of abdominal peripheral nerves were examined at a low stimulation frequency (1 Hz). Recording sites were marked with Fast Green FCF dye, which was electrophoretically injected through the recording micro-electrode. abdominal motoneurons. Extracellular spikes were recorded from single Renshaw cells. $T$ thoracic, $L$ lumbar, $b r$ branch, $E O m$ external oblique muscle, Stim stimulation, Ia-EPSP excitatory postsynaptic potential of Ia fibers, $R$-IPSP recurrent inhibitory postsynaptic potential

\section{Data analysis}

At the end of each experiment, animals were sacrificed via intravenous injection of a lethal dose of sodium pentobarbital. Animals were then perfused with $10 \%$ formalin solution administered via the aorta. The length of each peripheral nerve between the electrode and the point of entrance at the ventral roots was measured in order to calculate conduction velocity. Dyed regions were histologically examined in $50 \mu \mathrm{m}$ serial sections stained with cresyl violet. All data were saved on a digital system (PC-208AX, Sony, Japan). After the experiments, the data were digitized at $10 \mathrm{kHz}$ (Micro1401mkII, Cambridge Electronic Design, Cambridge, UK) for analyses with Spike 2 software (Cambridge Electronic Design, Cambridge, UK).

\section{Results}

\section{Monosynaptic excitation of abdominal motoneurons by afferent fibers}

Motoneurons were identified by searching for antidromic field potentials evoked by stimulation of the lateral and medial branches. Independent stimulation of either the lateral or medial branch enabled identification of the impaled motoneurons of the EO muscle, as well as other motoneurons of the IO, TA, and RA muscles.

Following the recording of antidromic spikes, we investigated synaptic potentials exerted on motoneurons by 
stimulating afferent fibers of the lateral and medial branches. Six of eight motoneurons analyzed exhibited antidromic spikes following stimulation of the medial branches, while two exhibited antidromic spikes following stimulation of the lateral branches. Membrane potential values ranged from -42 to $-68 \mathrm{mV}$, with a mean of approximately $-59 \mathrm{mV}$. The heights of the antidromic spikes ranged from 17 to $50 \mathrm{mV}$, with a mean of approximately $34 \mathrm{mV}$. Measurements of after-hyperpolarization were obtained between the point at which the post-spike potential dropped below the baseline level and the point of subsequent re-crossing. The duration of the after-hyperpolarization ranged from 100 to $155 \mathrm{~ms}$, with a mean of approximately $126 \mathrm{~ms}$. Typical recordings of the antidromic spike evoked by stimulating the medial branch and the after-hyperpolarization are shown in Fig. 2a.

We then investigated the reflex actions exerted on motoneurons by medial branch afferent fibers. Stimulation of the medial branch below the threshold of the motor axons at an intensity adequate for exciting only the lowest threshold afferent fibers evoked Ia-EPSPs in IO, TA, or RA muscle motoneurons, as illustrated in the upper trace of Fig. 2b. Figure $2 \mathrm{c}$ shows the relationship of the amplitude of an action a
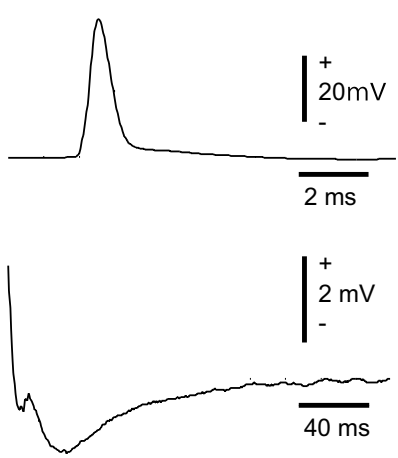

d
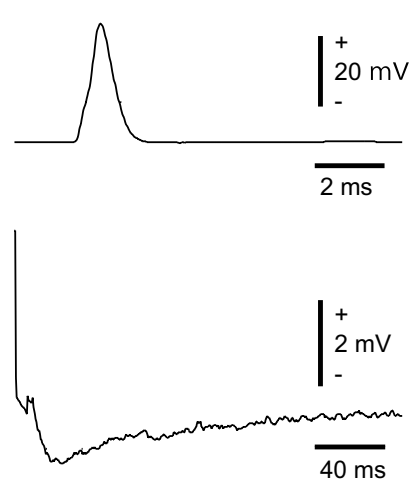

b
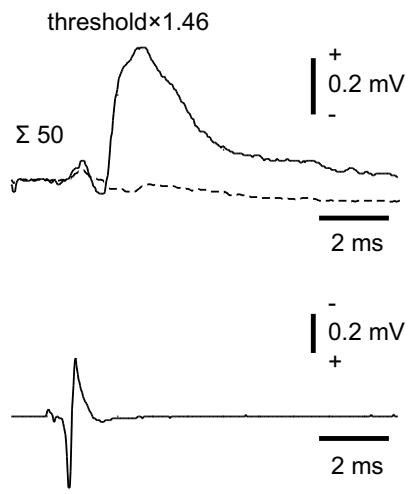

e
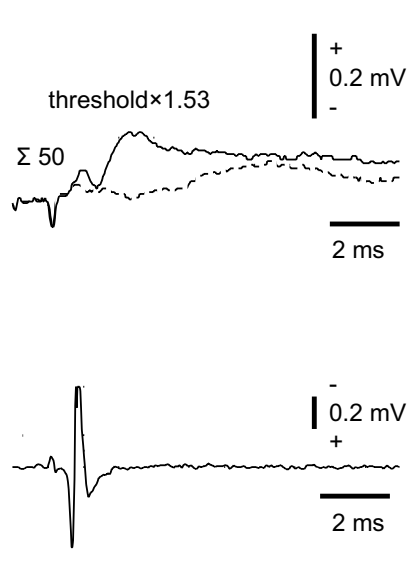

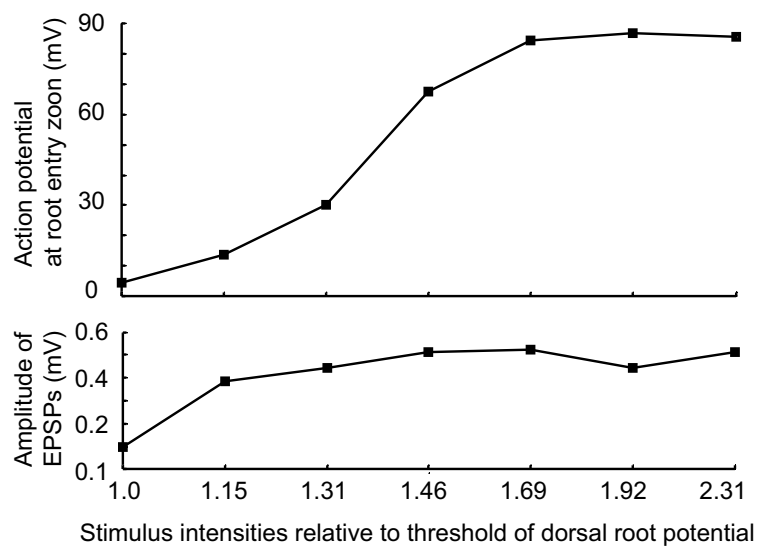

f
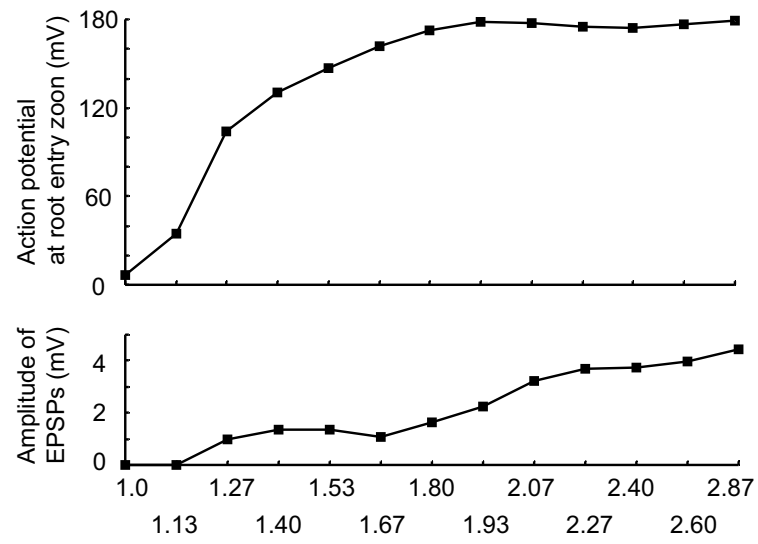

Stimulus intensities relative to threshold of dorsal root potential
Fig. 2 Reflex actions exerted on abdominal motoneurons by stimulating afferent fibers. a Antidromic spike evoked by stimulating the medial branch (upper trace) and after-hyperpolarization (lower trace). b Ia-EPSPs evoked by stimulating the medial branch below the threshold (upper trace) and cord dorsum potential (lower trace). The broken line indicates the extracellular recording from the impaled motoneuron. c Relationship of the amplitude of the action potential recorded at the root entry zone to the stimulus intensity (upper trace), and of the Ia-EPSP amplitude to stimulus intensity (lower trace). The abscissa indicates the stimulus intensities relative to the threshold of action potentials recorded at the root entry zone. The ordinate indicates the amplitude. d Antidromic spike evoked by stimulating the lateral branch (upper trace) and after-hyperpolarization (lower trace). e Ia-EPSPs evoked by stimulating the medial branch below the threshold (upper trace) and action potentials recorded at the root entry zone (lower trace). The broken line indicates the extracellular recording from the impaled motoneuron. f Relationship of the amplitude of the action potential recorded at the root entry zone to the stimulus intensity (upper trace), and of the Ia-EPSP amplitude to the stimulus intensity (lower trace). The abscissa indicates the stimulus intensities relative to the threshold of action potentials recorded at root entry zone. The ordinate indicates the amplitude. Ia-EPSP excitatory postsynaptic potential of Ia fibers; $R$-IPSP recurrent inhibitory postsynaptic potential 
potential recorded at the root entry zone and the amplitude of the Ia-EPSP to the stimulus intensity. When the intensity of stimulus was increased, a compound dorsal potential was observed. Although it was difficult to distinguish Ia and Ib action potentials recorded at the root entry zone, the amplitude of the action potential recorded at the root entry zone of lower threshold components exhibited maximal values when the intensity of stimulus was 1.69 times the threshold. Thus, a stimulus intensity of 1.69 times the threshold was thought to induce a maximal Ia response in motoneurons.

Five of eight motoneurons analyzed exhibited Ia-EPSPs following stimulation of the same nerve. As one motoneuron had a low threshold for antidromic excitation, no Ia-EPSPs could be recorded, while the remaining two motoneurons could not be analyzed. The latencies of Ia-EPSP onset, as measured from the summit of the initial positivity of the action potential recorded at the root entry zone, ranged from 1.0 to $1.6 \mathrm{~ms}$, with a mean value of $1.2 \mathrm{~ms}$. Ia-EPSP amplitudes ranged from 1.0 to $1.1 \mathrm{mV}$, with a mean value of $0.4 \mathrm{mV}$. Only one of five motoneurons exhibited Ia-EPSPs following stimulation of the synergic nerve (Fig. 2d, e). The latency of Ia-EPSP onset was $0.8 \mathrm{~ms}$. The amplitude of the Ia-EPSP was $0.1 \mathrm{mV}$ at a stimulus intensity of 1.67 times the threshold. Figure $2 \mathrm{f}$ shows the relationship between the amplitude of the action potential recorded at the root entry zone and the amplitude of the Ia-EPSPs to the stimulus intensity.

We then examined the three abdominal motoneurons in which Ia-EPSPs were evoked by stimulation of the L1 nerve in order to determine whether there was input from other spinal segments (T13, L2, and L3). None of these motoneurons exhibited any responses within the range of stimulus intensities that provided the maximal Ia response.

\section{R-IPSPs}

Stable intracellular recordings were obtained from 11 abdominal motoneurons after the ipsilateral T12-L4 dorsal roots had been cut. Figure 3 a shows the antidromic activation of EO motoneurons following stimulation of the L2 lateral branches. The resting membrane potentials ranged from -55 to $-70 \mathrm{mV}$, while conduction velocities ranged from 45 to $58 \mathrm{~m} / \mathrm{s}$. The stimulus voltage was gradually decreased until antidromic activation of impaled motoneurons could no longer be elicited, and membrane potentials were summed by triggering stimulation. Signal averaging revealed that R-IPSPs had occurred in one of 11 motoneurons (Fig. 3b), with an amplitude of $100 \mu \mathrm{V}$ and latency of $3.1 \mathrm{~ms}$. The duration of the R-IPSPs was approximately $12 \mathrm{~ms}$. Due to the presence of extracellular antidromic field potentials, subtraction of an extracellular recording is required to show the true shape of R-IPSPs and to measure latency. In this case, the latency from the stimulus was approximately $3.1 \mathrm{~ms}$, while that from the start of the antidromic field potential was $1.2 \mathrm{~ms}$. These latency values correspond to those for di-synaptic IPSPs evoked by collaterals of the alpha motor axons of abdominal motoneurons.

The hyperpolarizing shifts of the membrane potentials were presumed to represent a true transmembrane potential change (i.e., R-IPSPs), as they were absent in the extracellular control averages, the dorsal roots in the relevant spinal segments had been cut, and the effects could not be elicited through the primary afferents. Three of 11 abdominal motoneurons had a low threshold for antidromic activation, and thus could not be examined for hyperpolarizing potentials. The remaining seven abdominal motoneurons did not exhibit hyperpolarizing potentials when the stimulus was applied at a voltage just under that required for antidromic activation of the impaled motoneurons.

Intracellular recordings were obtained from some Renshaw cell-like neurons in the ventral horn and action potentials in their high-frequency discharge patterns were observed following stimulation of the abdominal nerves (Fig. 3c). Latency values ranged from 2.4 to $2.5 \mathrm{~ms}$ from the onset of the stimulus. At each stimulus intensity, amplitude values ranged from 9 to $11 \mathrm{mV}$. Latency tended to decrease as the intensity of the stimulus increased, and two to three spikes were observed for each stimulus.

\section{Extracellular recording from Renshaw cells}

We attempted to obtain extracellular recordings from Renshaw cells in the ventral horn of the upper lumbar segments (L1-L2). Bipolar cuff electrodes were placed in the proximal portion of the L1-L2 nerves for electrical stimulation. Renshaw cells were identified based on orthodromic activation following stimulation of abdominal nerves. Recordings were obtained from Renshaw cells just above the region in which maximal antidromic field potentials were obtained following stimulation of abdominal nerves. Short response latencies were observed for 13 cells following abdominal nerve stimulation. Figure 4a shows a typical example of Renshaw cell activity following stimulation of the L1 abdominal nerve, in which the maximum discharge was associated with two to three spikes per stimulus. Responses became stronger, while latency values decreased, as the intensity of the stimulus increased with maximal stimulation, producing two to three spikes per stimulus. The cell depicted in Fig. 4a responded at a frequency of approximately $800 \mathrm{~Hz}$ when stimulation was applied at 1.05 times the threshold. Latencies for the first spikes ranged from 1.5 to $2.5 \mathrm{~ms}$, suggesting that spikes were activated di-synaptically. Figure $4 \mathrm{~b}$ depicts findings from simultaneous recording of Renshaw cell activity and the antidromic field potentials. When the stimulus intensity was increased, Renshaw cells fired at the same stimulus intensity in which the antidromic filed 
a
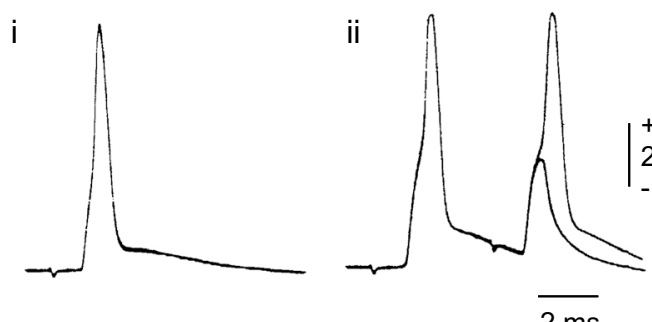

$2 \mathrm{~ms}$

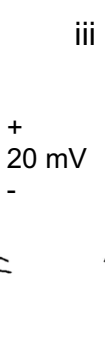

iii

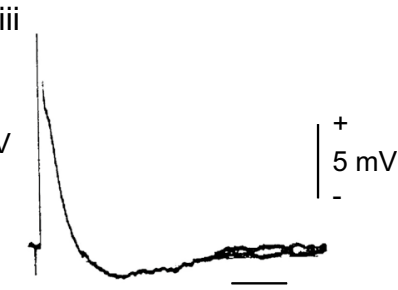

$\overline{10 \mathrm{~ms}}$

b

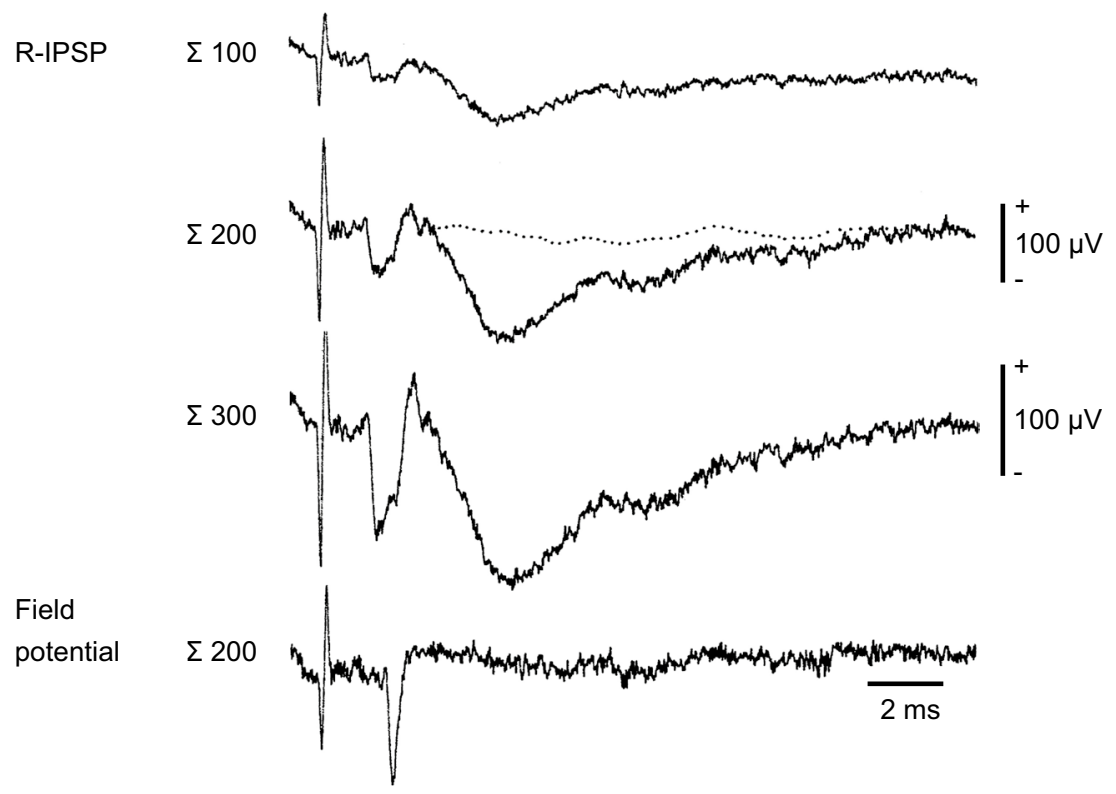

C

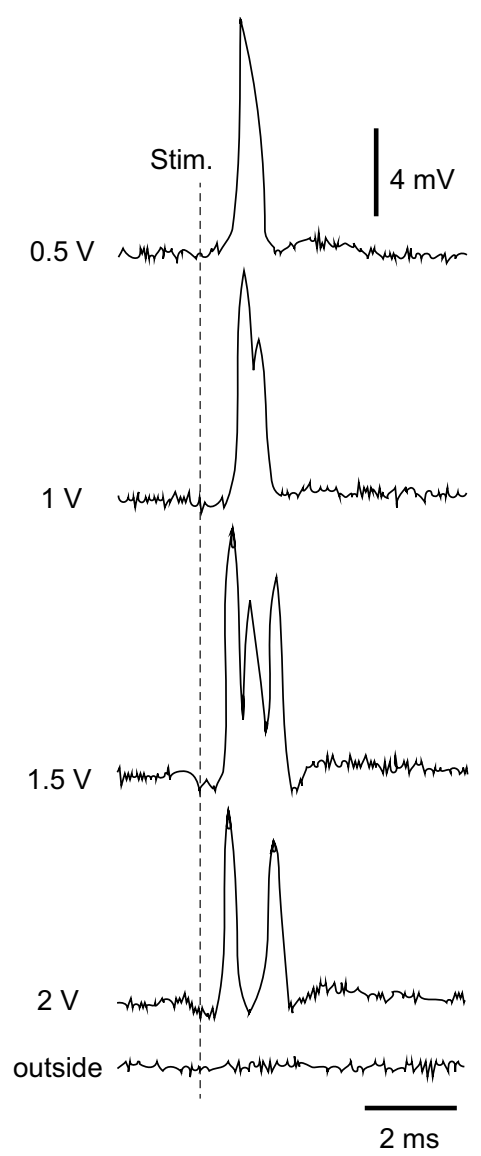

Fig. 3 Antidromic spikes and R-IPSPs of abdominal motoneurons. a Antidromic spikes (i) and response to short interval double stimuli (ii) and after hyperpolarization (iii). b R-IPSPs following stimulation of the EO nerve at an intensity just below the threshold for activation of antidromic spikes in the impaled motoneuron (average values). The broken line indicates the extracellular recording from the impaled motoneuron. c Intracellular recordings from Renshaw cells following the application of different stimulus intensities to the L2 nerve (L2 abdominal medial and lateral branches). The bottom line indicates the extracellular field potential just outside of the impaled cell. $R$-IPSP recurrent inhibitory postsynaptic potential

location of the abdominal motor nucleus and the recording sites that projected to the lumbar transverse section $(N=7$, six at L1; $N=1$ at L2). Recording sites were observed dorsal and medial to the abdominal motor nucleus. The recording site of this Renshaw cell was located in the region just dorsal to abdominal nucleus. Figure $4 \mathrm{f}$ represents recording sites marked with Fast Green FCF dye.

\section{Discussion}

In the present study, we obtained intracellular and extracellular recordings from abdominal motoneurons and Renshaw cells, respectively, in order to examine monosynaptic excitation from Ia fibers, recurrent inhibition from Renshaw cells, and Renshaw cell behavior in the upper lumbar segments. IaEPSPs were elicited in five of eight motoneurons following 
a

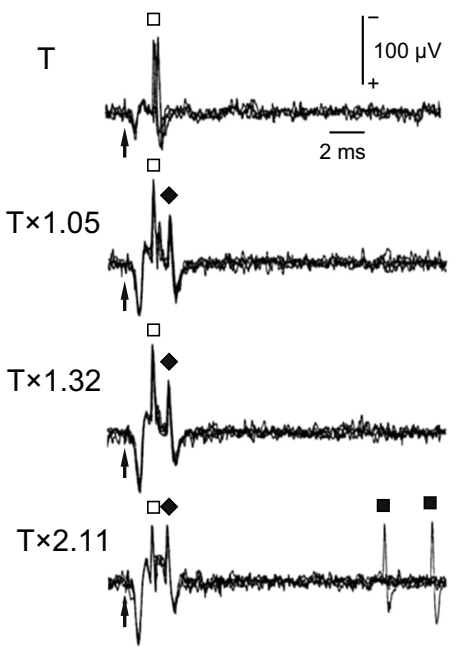

b
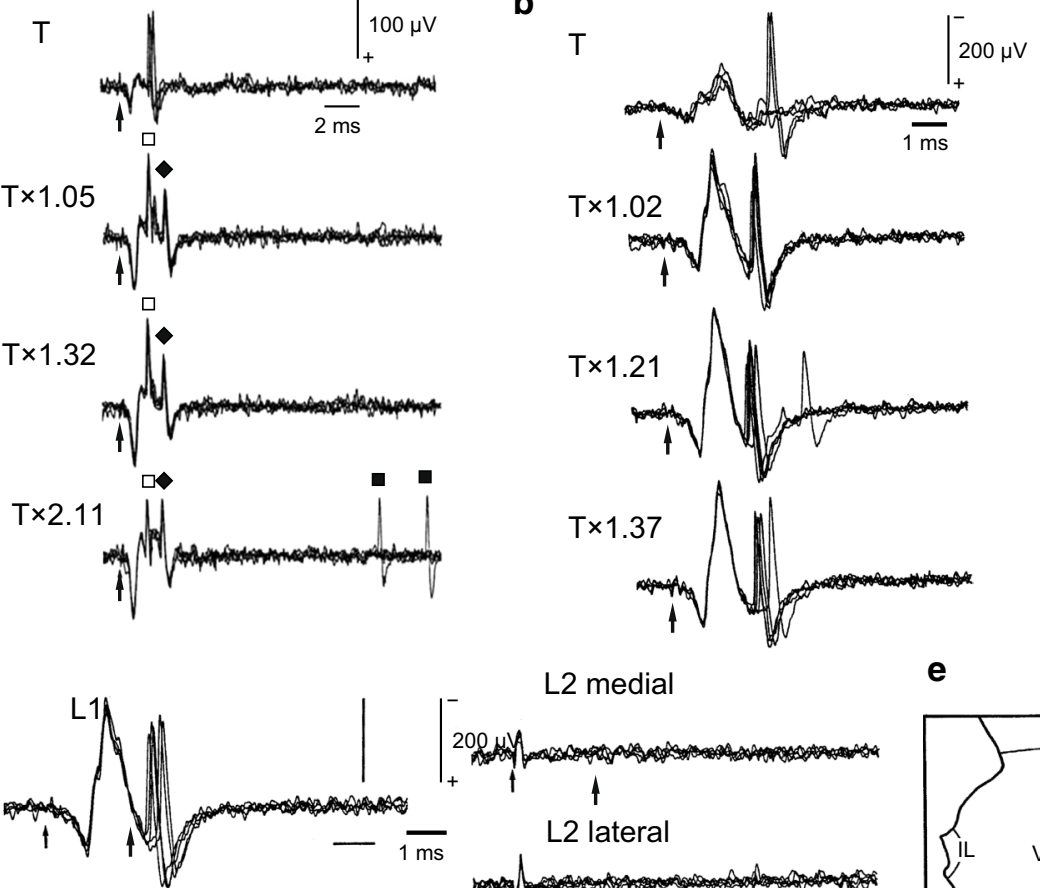

$\int_{1}^{T 13}$

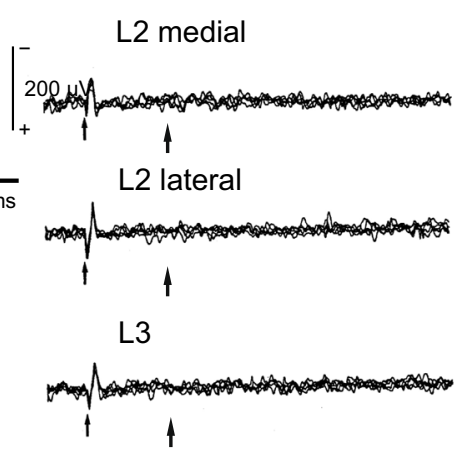

L2 medial

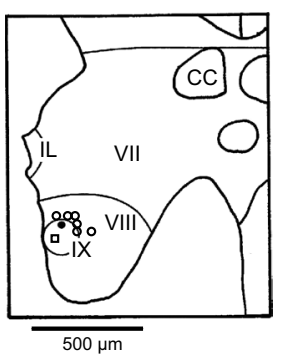

C

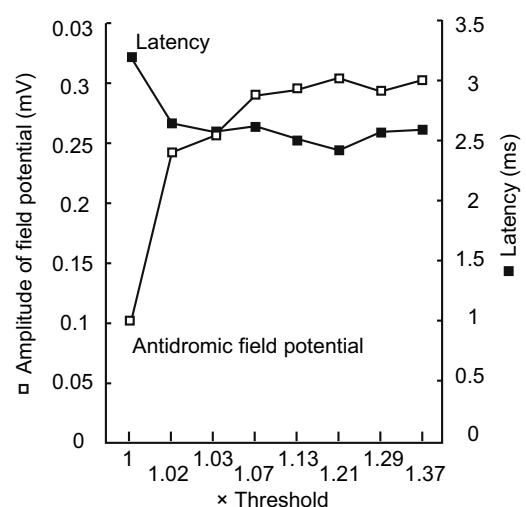

f

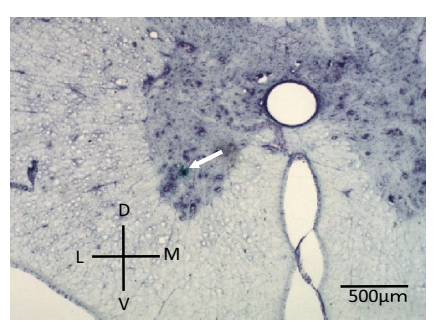

Fig. 4 Representative response of Renshaw cells in the ventral horn. a Orthodromic activation following stimulation of the L1 nerve (L1 abdominal medial and lateral branches). The intensity of electrical stimulation was gradually increased from threshold $(T)$ to $T \times 2.11$. Each panel reflects the results of five superimposed traces. Open squares, filled diamonds, and filled squares represent the first, second, and third spikes, respectively. Arrows indicate the time of stimulation. b Synaptic excitation following stimulation of the L1 nerve. Arrows indicate the time of stimulation. c Relationship among stimulus voltage, latency of the activated spikes, and the amplitude of the field potentials. The ordinate indicates the amplitude of the field potential (left) and the latency of the activated spikes (right). Open squares represent the antidromic field potential. Filled squares represent the latency of the antidromic spikes. The abscissa indicates the intensity

stimulation of the same nerve. Ia-EPSPs were limited to the same muscle and spinal segment. Renshaw cells fired highfrequency bursts at a short latency following stimulation and responded to stimulation at the same spinal segment only. R-IPSPs were elicited in one of 11 abdominal motoneurons following stimulation at an intensity subthreshold to that required for activation of impaled motoneurons.

\section{la-EPSPs}

Physiological studies in the hind limb and intercostal muscles have revealed that Ia fibers convey monosynaptic excitation to motoneurons of the same and synergic of the stimulation multiplied by the threshold of the stimulus voltage. d Convergence of excitatory inputs from other spinal segments. L2 medial: L2 medial branch. L2 lateral: L2 lateral branch. The T13, L1, and L3 nerves were stimulated. Note that Renshaw cells were activated only when the L1 nerve was stimulated. e Cross-sectional schematics of the spinal cord at the representative L1 spinal segment. The open square indicates the abdominal motor nucleus. The filled circle represents the Renshaw cell recording sites. Open circles represent the recording sites that projected to the lumber transverse section. Roman numerals indicate the Rexed laminae. $I L$ nucleus intermedio-lateralis; CC Clarke's column. f Recording sites of Renshaw cells. Cross-section of the spinal cord at the L1 spinal segment. White arrow represents recording sites marked with Fast Green FCF dye

muscles, and that they are responsible for almost all of the monosynaptic EPSPs to motoneurons of the relevant muscles [8-11]. Morphological studies in the hind limb [19] and intercostal muscles [20] have also demonstrated that the intramedullary trajectories of single Ia afferent fibers exhibit connections with motoneurons. In one previous study, afferent volleys were divided into " $1 \mathrm{a}$ ", " $1 \mathrm{~b}$ ", and " $1 \mathrm{c}$ " components based on increases in stimulus intensity in the thoracic spinal cord, and the authors reported that the maximal monosynaptic EPSP to the 1a component was 1.8 times the threshold [11]. In the present study, afferent volleys could not be clearly divided into 
Ia and Ib components, and maximal Ia-EPSP amplitudes were observed at a stimulus intensity of 1.69 times the threshold.

In the present study, five of six abdominal motoneurons examined exhibited Ia-EPSPs following stimulation of the same nerve. However, four of five motoneurons examined did not exhibit Ia-EPSPs following stimulation of the synergic nerve. In addition, no abdominal motoneuron responses were observed following stimulation of other segments. Previous research has indicated that the Ia fibers of respiratory muscle nerves provide monosynaptic excitation to the synergic motoneurons of the adjacent segments [20, 21]. In contrast to those findings, our results indicated that the IaEPSPs of abdominal motoneurons are limited to the same muscle and spinal segment.

Our results revealed that the duration of after-hyperpolarization was approximately $126 \mathrm{~ms}$, which is greater than the maximal value reported for intercostal motoneurons (range, 65-110 ms) [11]. Based on previous findings for hind limb motoneurons, these values are considered indicative of slow responses (cutoff, $110 \mathrm{~ms}$ ) [22]. Latency to Ia-EPSP onset from the summit of the initial positivity of the action potential recorded at the root entry zone ranged from 1.0 to $1.6 \mathrm{~ms}$ (mean, $1.2 \mathrm{~ms}$ ). These values are longer than those reported for the hind limb and intercostal muscles $(0.5-0.8 \mathrm{~ms})$ [ 10 , 11]. These findings suggest that the Ia fibers of the abdominal muscles decrease in size upon entry into gray matter of the spinal cord and Ia-EPSPs are monosynaptic. However, it cannot be excluded that Ia-EPSPs with long latency are di-synaptic EPSP.

\section{Recurrent inhibition by Renshaw cells}

Motoneurons innervating the hind limb and fore limb muscles in the cat exhibit strong recurrent inhibition pathways, which become weaker as one moves from the proximal to distal muscles [23, 24]. Recurrent inhibition is also abundant in the motoneurons innervating the muscles of the back and neck in cats [15, 25]. However, previous studies have indicated that respiratory motoneurons (e.g., phrenic and intercostal motoneurons) exhibit weak recurrent inhibition $[11,16,26]$. Our electrophysiological experiments identified recurrent inhibition (R-IPSPs) in only one of 11 abdominal motoneurons examined. These results were likely due to an underestimation of the stimulus conditions required for peripheral nerves, as we adjusted the stimulus strength to remain subthreshold to that for the axons of impaled motoneurons. Indeed, two EO motoneurons exhibited a low threshold for excitation, and R-IPSPs could not be examined in these motoneurons. These findings suggest that only a small proportion of abdominal motoneurons receive recurrent inhibition.
Synaptic activation was observed in single Renshaw cells only when abdominal nerves of the same segment were stimulated, suggesting that the extent of recurrent inhibition/RIPSPs is limited to the same spinal segment. This finding is in contrast to those regarding recurrent inhibition in intercostal motoneurons, which can be observed up to three segments away from the stimulated segment [14]. Short duration of repetitive spike activity occurred for Renshaw cells associated with abdominal nerve stimulation, similar to findings observed for intercostal motoneurons [11, 14], and in contrast to those for hind limb [13] and fore limb motoneurons [24].

The recording sites of Renshaw cells were located in spinal regions dorsal and medial to the abdominal motor nucleus. However, it was difficult to identify Renshaw cells in the regions in which maximal antidromic field potentials were observed, since large antidromic field potentials make it difficult to discriminate individual Renshaw cell spikes. Thus, some Renshaw cells may be located in the abdominal motor nucleus. Renshaw cells are located in the medio-ventral part of the hind limb motor nucleus $[27,28]$ and in the ventral border of the ventral horn of the intercostal nucleus [14]. Previous studies have revealed that Renshaw cells lie close to the phrenic motor nucleus [26, 29], suggesting that the location of Renshaw cells differs among various nuclei.

\section{Physiological roles of la-EPSPs and R-IPSPs in the abdominal muscles}

Ia excitation pathway to motoneurons and inhibitory pathway to motoneurons of antagonist muscle are important neuronal connections of hindlimb. Ia inhibitory interneurons lie between Ia afferents and motoneurons of antagonist muscle. Previous studies have investigated reflex actions from different types of primary afferents to motoneurons, demonstrating that conditioning ventral root stimulation effectively depresses transmission to motoneurons in Ia inhibitory pathways, and that such depression is caused by postsynaptic inhibition of the interposed interneurons [31-33]. Renshaw cells inhibit motoneurons and Ia inhibitory interneuron of the antagonist muscle. Previous research has also suggested that recurrent inhibition serves as variable gain regulator for motor output [30].

The abdominal muscles exhibit multi-segmental innervation from the thoracic to lumbar spinal segments and relate with various movements. The activities of intercostal muscles and back muscles may correlate with the abdominal muscles, but the agonists and antagonists of the abdominal muscles cannot be identified, since these muscles change from moment to moment during movements. In the present study, the Ia-EPSPs and R-IPSPs existed in abdominal motoneurons, but synaptic effects were limited to the same spinal segment and weak, suggesting that the motor output of the 
abdominal muscles is controlled at the segmental level. Ia inhibitory interneurons are important in hindlimb muscle, since the function of muscles is the flexion and extension of joints, although agonists and antagonists many times show co-contraction. Neither such reciprocal inhibition of antagonistic motoneurons have been identified in the abdominal muscles, nor the intercostal muscles. The strong Renshaw effect and Ia reciprocal inhibition does not seem to be an important synaptic effect on the muscles such as abdominal muscles that have not stereotype movements but have various functions. Thus, the integrated control among spinal levels may involve additional, interconnected networks within the spinal cord and upper central nervous system.

The present study possesses some limitations of note. First, R-IPSPs were elicited in one of 11 abdominal motoneurons following stimulation at an intensity subthreshold to that required for activation of impaled motoneurons, but this number is very small compared to the number of Renshaw cells from which extracellular recordings were obtained. Although it is possible that Renshaw cells did not reach the axons of the motoneurons receiving their input, we were unable to verify this matter in the present study. Second, we did not examine interactions between the abdominal and back/intercostal muscles, especially those involving the interposed interneurons. Therefore, we were unable to determine the relationship between the abdominal muscles and the synergistic or antagonistic muscles, or the effects of excitation/inhibition caused by interneurons. Third, all experiments were performed with the subjects under anesthesia, and it remains unknown how Renshaw cells behave during wakefulness. However, the abdominal muscles reflect respiratory function even under anesthesia, and are likely to exhibit similar behavior during wakefulness.

\section{Conclusions}

Intracellular recordings were obtained from the abdominal motoneurons of the upper lumbar segments (L1-L2) in cats. When muscle nerves were electrically stimulated to activate sensory afferents with dorsal roots left intact, Ia-EPSPs were elicited in five of six motoneurons following stimulation of the same nerve and limited to the same muscle and spinal segment. When muscle nerves were electrically stimulated to activate motor axons whose dorsal roots had been sectioned, Renshaw cells in the ventral horn fired highfrequency bursts at a short latency following stimulation and responded to stimulation at the same spinal segment only. R-IPSPs were elicited in one of 11 abdominal motoneurons following stimulation at an intensity subthreshold to that required for activation of impaled motoneurons, suggesting that only a small proportion of abdominal motoneurons receive recurrent inhibition. Further studies are required to examine the roles of the abdominal muscles as synergists or antagonists in relation to the back muscles.

Acknowledgements This study was supported by a KAKENHI grant (25350621) from the Japan Society for the Promotion of Science. We would also like to thank Editage (www.editage.jp) for English language editing.

Author contributions All co-authors participated in data collection and analysis. All co-authors have read and approved the final manuscript.

\section{Compliance with ethical standards}

Conflict of interest The authors declare that they have no conflicts of interest.

Ethical approval All experimental procedures were approved by the Animal Ethics Committee of Ibaraki Prefectural University of Health Sciences and were in accordance with the guiding principles for care and use of animals in the field of physiological sciences outlined by the Physiological Society of Japan.

\section{References}

1. Niwa M, Muramatsu K, Sasaki S-I (2015) Discharge patterns of abdominal and pudendal nerves during induced defecation in anesthetized cats. J Physiol Sci 65:223-231

2. Waters RL, Morris JM (1972) Electrical activity of muscles of the trunk during walking. J Anat 111:191-199

3. De Troyer A (1983) Mechanical role of the abdominal muscles in relation to posture. Respir Physiol 53:341-353

4. Miller AD, Tan LK, Suzuk I (1987) Control of abdominal and expiratory intercostal muscle activity during vomiting: role of ventral respiratory group expiratory neurons. J Neurophysiol 57:1854-1866

5. Bishop B (1964) Reflex control of abdominal muscles during positive pressure breathing. J Appl Physiol 19:224-232

6. Russel JA, Beverly P, Bishop BP, Hyatt RE (1987) Discharge of abdominal muscle $\alpha$ and $\gamma$ motoneurons during expiratory loading in cats. Exp Neurol 97:179-192

7. Niwa M, Nakayama K, Sasaki S-I (2008) Morphological study of external oblique motor nerves and nuclei in cats. Anat Sci International 83:17-25

8. Lloyd DPC (1943) Conduction and synaptic transmission of the reflex response to stretch in spinal cats. J Neurophysiol 6:317-326

9. Lloyd DPC (1943) Reflex action in relation pattern and peripheral source of afferent stimulation. J Neurophysiol 6:111-119

10. Eccles JC, Eccles RM, Lundberg A (1957) Synaptic actions on motoneurones in relation to the two components of the group I muscle afferent volley. J Physiol 136:527-546

11. Sears TA (1964) Some properties and reflex connections of respiratory motoneurones of the cat's thoracic spinal cord. J Physiol 175:386-403

12. Eccles JC, Fatt P, Koketsu K (1954) Cholinergic and inhibitory synapses in a pathway from motor-axon collaterals to motoneurons. J Physiol 126:524-562

13. Eccles JC, Eccles RM, Iggo A, Lundberg A (1961) Electrophysiological investigations of Renshaw cells. J Physiol 159:461-478

14. Kirkwood PA, Sears TA, Westgaard RH (1981) Recurrent inhibition of intercostal motoneurons in the cats. J Physiol 319:111-130

15. Brink EE, Suzuki I (1987) Recurrent inhibitory connexions among neck motoneurones in the cat. J Physiol 383:301-326 
16. Hilaire G, Khatib M, Monteau R (1986) Central drive on Renshaw cells coupled with phrenic motoneurons. Brain Res 376:133-139

17. Gill PK, Kuno M (1963) Excitatory and inhibitory actions on phrenic motoneurones. J Physiol 168:274-289

18. Uga M, Niwa M, Ochiai N, Sasaki S-I (2010) Activity pattern of the diaphragm during voluntary movements in awake cats. $\mathrm{J}$ Physiol Sci 60:173-180

19. Ishizuka N, Mannen H, Hongo T, Sasaki S (1979) Trajectory of group Ia afferent fibres stained with horseradish peroxidase in the lumbosacral spinal cord of the cat: three-dimensional reconstructions from serial sections. J Comp Neurol 186:189-211

20. Nakayama K, Niwa M, Sasaki S-I, Ichikawa T, Hirai N (1998) Morphology of single primary spindle afferents of the intercostal muscles in the cat. J Comp Neurol 398:459-472

21. Eccles RM, Sears TA, Shealy CN (1962) Intra-cellular recording from respiratory motoneurones of the thoracic spinal cord of the cat. Nature 193:844-846

22. Eccles JC, Eccles RM, Lundberg A (1958) The action potentials of the alpha motoneurones supplying fast and slow muscles. J Physiol 142:275-291

23. McCurdy ML, Hamm T (1992) Recurrent collaterals of motoneurons projecting to distal muscles in the cat hindlimb. J Neurophysiol 67:1359-1366

24. Illert M, Wietelmann D (1989) Distribution of recurrent inhibition in the cat forelimb. Prog Brain Res 80:273-281
25. Jankowska E, Odutola A (1980) Crosses and uncrossed synaptic actions on motoneurones of back muscles in the cat. Brain Res 194:65-78

26. Lipski J, Fyffe REW, Jodkowski J (1985) Recurrent inhibition of cat phrenic motoneurons. J Neurosci 5:1545-1555

27. Jankowska E, Lindström S (1971) Morphological identification of Renshaw cells. Acta Physiol Scand 81:428-430

28. Cullhem S, Kellerth J-O (1978) A morphological study of the axons and recurrent axon collaterals of cat sciatic a-motoneurons after intracellular staining with horseradish peroxidase. J Comp Neurol 178:537-558

29. Hilaire G, Khatib M, Monteau R (1983) Spontaneous respiratory activity of phrenic and intercostal Renshaw cells. Neurosci Lett 43:97-101

30. Hultborn H, Lindstrom S, Wigstrom H (1979) On the function of recurrent inhibition in the spinal cord. Exp Brain Res 37:399-403

31. Hultborn H, Jankowska E, Lindstrom S (1971) Recurrent inhibition from motor axon collaterals of transmission in the Ia inhibitory pathway to motoneurones. J Physiol 215:591-612

32. Hultborn H, Jankowska E, Lindstrom S (1971) Recurrent inhibition of interneurones monosynaptically activated from group Ia afferents. J Physiol 215:613-636

33. Burke RE, Fedina L, Lundberg A (1971) Spatial synaptic distribution of recurrent and group Ia inhibitory systems in cat spinal motoneurones. J Physiol 214:305-326 